\title{
Analysis of Cases ofCervical Cancer in Pregnant Women
}

\author{
Di Bo ${ }^{1, *}$ \\ Jing Liu' ${ }^{1} *$ \\ Yuhong $\mathrm{Li}^{2}$ \\ Jiandong Wang ${ }^{3}$ \\ Yudong Wang (1D) ${ }^{2}$
}

'Perinatal Department of Obstetrics, Beijing Obstetrics and Gynecology Hospital, Capital Medical University, Beijing Maternal and Child Health Care Hospital, Beijing, 100026, People's Republic of China; ${ }^{2}$ Department of Gynecologic Oncology, International Peace Maternity and Child Health Hospital, Shanghai Jiao Tong University School of Medicine, Shanghai, 200030, People's Republic of China; ${ }^{3}$ Department of Gynecologic Oncology, Beijing Obstetrics and Gynecology Hospital, Capital Medical University, Beijing Maternal and Child Health Care Hospital, Beijing, 100026, People's Republic of China

*These authors contributed equally to this work
Correspondence: Jiandong Wang Department of Gynecologic Oncology, Beijing Obstetrics and Gynecology Hospital, Capital Medical University, Beijing Maternal and Child Health Care Hospital, No. 25I Yaojiayuan Road, Chaoyang District, Beijing, 100026, People's Republic of China

Tel +86/39l0566755

Email wangjiandongxy@ccmu.edu.cn

Yudong Wang

Department of Gynecologic Oncology, International Peace Maternity and Child Health Hospital, Shanghai Jiao Tong

University School of Medicine, NO. I96I,

Huashan Road, Shanghai, 200030, People's

Republic of China

Tel +86I80I73I6053

Email gfywangyudong@126.com
Purpose: To analyze the clinical characteristics and prognosis of pregnancies that are complicated by cervical cancer and to identify appropriate therapeutic protocols.

Patients and Methods: Clinical data were acquired from 14 subjects attending hospitals in Beijing and Shanghai, China, between January 2015 and December 2019. Then, we analyzed clinical characteristics, diagnosis, treatments, and prognosis.

Results: Of the 14 cases, there were two cases of abortion, two cases of chemotherapy during the second trimester, two cases of cold knife conization, and eight cases of expectant management and extended postpartum treatment. The gestational age ranged from 28 to 40 weeks. All subjects and infants survived except for the fetuses that underwent abortions.

Conclusion: Early diagnosis of cervical therapy, and the provision of multivariate therapy, can prolong gestational age and improve the survival rate of both pregnant women and infants.

Keywords: pregnancy, cervical cancer, treatment, pregnancy outcome

\section{Introduction}

Cervical cancer is the second most common malignant tumor in Chinese females. This is a common gynecological malignancy, which predominantly affects females aged between 20 and 40 years-of-age. Cervical cancer in pregnancy is much rarer than in non-pregnant females. Previous research estimated that the incidence of cervical cancer in pregnancy lay between $1 / 2000$ and $1 / 10,000 .{ }^{1}$ Another study, carried out in China, estimated that the incidence of cervical cancer in pregnancy was $0.10-0.1 \%{ }^{2}$ Due to the special immune status of pregnancy, the presence of cervical cancer during pregnancy will not affect maternal and fetal outcomes during the middle and late stages of pregnancy. Therefore, over recent years, there has been some controversy relating to whether pregnant females with cervical cancer require the immediate termination of pregnancy. However, following the identification of cervical cancer, a significant number of pregnant women still wish to continue their pregnancy. Therefore, there is a clear need to improve the quality of life for women with cervical cancer during pregnancy. Consequently, there is a need to develop differential diagnoses and treatment strategies according to the period, age, and pregnancy requirements. Over the last five years, there has been a significant increase in the widespread use of screening protocols for cervical cancer; this has led to a significant reduction in the incidence of cervical cancer within the screened population; ${ }^{3}$ these effects are also related to an increased awareness of cervical screening and an increase in the rate of vaccination against 
HPV. According to the national status of China, the Chinese Society of Obstetrics and Gynecology has designated relevant guidelines for the screening of cervical cancer during pregnancy: women who have not participated in cervical cancer screening, especially those who have never been screened, and women who need to be rescreened for cervical cancer; the guidelines also state that cervical cancer screening should be performed during prepregnancy examinations or at the first prenatal examination. A screening method based on cervical cytology will not threaten the mother or infant at any point during the entire pregnancy. Patients with cervical cancer, for whom clinical symptoms and signs cannot be excluded, should be directly referred for colposcopy or biopsy and diagnosed according to pathological results. Four patients classified as ASC-US following high-risk human papillomavirus (HPV) detection, negative cases should be subject to postpartum re-examination; positive patients should be referred (directly if necessary) to colposcopy. Patients classified as LSIL should be referred for colposcopy. If the cervical cytology is classified as ASC-US or LSIL and there is no suspicious clinical history or signs, then cervical cancer screening can be performed six weeks after delivery. If the initial colposcopy resulted in a classification of LSIL or histological LSIL (cin1), then postpartum re-examination should be recommended. Cytological classifications of ASC-H, HSIL, and above, AGC, and above should be referred for colposcopy. Beijing and Shanghai are both first-tier cities in China; as such, it is common for disease prevention and screening strategies to be implemented in these cities. The screening rate for the ThinPrep cytology test (TCT) in the Beijing Obstetrics Department is known to be 30.76\% (21951/ 71360); this compares to $100 \%$ in Shanghai. The Beijing Obstetrics and Gynecology Hospital and the Shanghai National Women's and Infants Hospital are both third class A hospitals, with advanced therapeutic experience.

In the present study, we retrospectively analyzed clinical data from 14 pregnancies (over the last five years) that were complicated by cervical lesions and managed by The Beijing Obstetrics and Gynecology Hospital and the Shanghai National Women's and Infants Hospital. For each pregnancy, we analyzed a range of clinical characteristics, examinations, and treatment methods. We also followed-up with each patient to determine the outcomes of their pregnancies. Further treatment and nursing care of patients with cervical cancer in a subsequent pregnancy used cytology test in obstetrics is $30.76 \%(21951 / 71360)$ in Beijing and $100 \%$ in Shanghai.

\section{Materials and Methods}

Data was retrospectively acquired from 152,116 cases of labor and delivery at Beijing Obstetrics and Gynecology Hospital and Shanghai Maternal and Child Hospital between January 2015 and December 2019. Between these dates, we identified 14 cases of pregnancy that had been complicated by cervical cancer; this relates to an incidence of $0.0078 \%(0.7889 / 10,000)$; this is lower than the previously reported rate of $0.01 \%(1 / 10,000) .{ }^{1}$ The mean age of the 14 pregnant women complicated by cervical cancer was $34.1 \pm 5.67$ years (range: 24-42 years). Excluding two cases that resulted in an abortion during the first and second trimesters, the mean gestational age was $35 \pm 9.28$ weeks. The severity of cervical cancer ranged from stage IA1 to IIB. During TCT examinations, the clinicians usually collected samples of cervical cells with a special sampler for TCT and then placed the sampler into a vial containing cell preservation solution and transferred to the clinical laboratory. This allowed the cells to be rinsed prior to downstream analysis. In the laboratory, samples were dispersed and filtered by an automatic cell detector to remove blood, mucus, and inflammatory tissue. In this way, a thin and wellpreserved layer of cells was acquired for microscopic detection and diagnosis. Patients receiving HPV examination were monitored for high-risk and low-risk HPV types, including their prenatal examination. The kits used were not identical.

\section{Diagnosis}

All pregnant women in Shanghai were screened for cervical cancer during their pregnancies. Furthermore, all pregnant women in Beijing who did not undergo pelvic examination within one year agreed to be re-evaluated by cervical cancer screening. All patients with a normal cervical appearance upon pelvic examination also underwent liquid-based cytology tests. Depending on their results, patients were either monitored regularly or given HPV tests and colposcopy. For patients with visible cauliflowershaped exophytic lesions on the cervix, we performed colposcopy-guided cervical biopsy and sent tissue samples for pathological examination. All diagnoses were based on pathological results. If necessary, immunohistochemical examination was performed to further differentiate between adenocarcinoma and small cell carcinoma. 


\section{Results}

As the economy and universal cervical cancer screenings develop, cervical cancer in pregnancy can be caught at earlier stages. All 14 subjects analyzed in the present study were at stage IIB or better at the time of diagnosis. None of the cases were classified as stage III-IV. The prognosis for a pregnancy that was complicated by cervical cancer, along with the rate of survival for puerperants and neonates improved coincidentally with an early diagnosis and effective chemotherapy and surgery during pregnancy.

\section{Clinical Manifestations}

There was no significant difference in terms of the clinical manifestations of cervical cancer when compared between pregnant and non-pregnant patients. Most patients of the patients showed no increase in secretion, abdominal pain, or vaginal drainage. However, four cases experienced repeat episodes of vaginal bleeding during pregnancy. A cervical vegetable tumor was detected during treatment; pathological analysis of a biopsy acquired by colposcopy suggested cervical cancer. Due to the state of pregnancy, the cause of vaginal bleeding can be complex and associated with several factors, including threatened abortion or placenta previa, thus delaying diagnosis. In the study, four patients with recurrent vaginal bleeding were examined by gynecologists and colposcopy-guided cervical biopsy, followed by pathological examination, was used to confirm the diagnosis of cervical cancer.

\section{Histopathological Analysis}

Small pieces of tissue were removed either from the cervical epithelium or directly from visible cervical lesions with biopsy forceps. These tissue samples were then embedded in paraffin and stained with hematoxylin-eosin staining. Immunohistochemical examination was performed if necessary.

Of the 14 cases pregnancies that were complicated by cervical cancer, there were two cases of adenocarcinoma, three cases of adenosquamous carcinoma, one case of endometrioid adenocarcinoma, and eight cases of cervical squamous cell carcinoma.

One of the two subjects with adenocarcinoma had already been diagnosed prior to her pregnancy. Her HPV test also showed a positive result for HPV 6 and 11. The other subject was seven-week pregnant when an exogenous tumor was found on her cervix. Further evaluation by biopsy indicated adenocarcinoma.
Of the three subjects with adenosquamous carcinoma, one had a cervical exogenous tumor and was biopsied in her 13th week of pregnancy. She was positive for all HPV strains except for strains 16, 18, and 33. Another patient had an exogenous tumor and underwent colposcopyguided cervical biopsy in her 18th week of pregnancy; this patient did not receive an HPV test. The final patient diagnosed with adenosquamous carcinoma was classified as LSIL result by cervical cytology screening and tested positive for all HPV strains except for strains 16, 18 and 33. However, a definitive diagnosis was not made until 5 months after delivery.

Only one subject had endometrial adenocarcinoma. This subject achieved a normal result in the cervical cytology test during early screening; however, a polyp-like neoplasm was noted prior to delivery. Further evaluation after cesarean section led to the final diagnosis.

Squamous cell carcinoma was the most common type of cancer detected in this study; eight subjects received this diagnosis. One HPV-positive subject had been diagnosed two years previously when she had her first child. Subsequently, she was treated by cold knife conization; all post-operative follow-ups were normal. This patient subsequently became pregnant and delivered a healthy baby. Two patients were transferred to our hospital for the evaluation of cervical cancer evaluation and treatment during their third trimester; both patients were HPV-16 positive. Two other subjects were diagnosed with cervical cancer after the delivery: one was HPV-16 positive, and the other did not undergo an HPV test, but was classified as LSIL following cervical cytology screening. Neither of these two subjects returned for follow-ups or biopsy while pregnant. The three remaining subjects were detected by routine prenatal examination and cervical cytology screening. All three of these subjects had stage IA1 cervical cancer at the time of diagnosis; two of these were HPV-16 positive. One of these subjects did not have an HPV test.

\section{Treatment and Pregnancy Outcome}

In the study, we identified four subjects with stage IA1 cervical cancer. The subject, who received cold knife conization two years previously, had stage IA1 cervical cancer. After treatment, she was able to become pregnant and give birth to a healthy baby by vaginal delivery during week 40 . Both the subject and the baby survived. Reactive cellular changes were noted in the cervical cytology test of one other patient with persistent HPV-16 infection. Postpartum colposcopy indicated stage IA1 cervical 
cancer. This patient received extensive laparoscopic cervical resection, plus pelvic lymph node resection. In one case, a stage IA1 squamous cell carcinoma of the cervix was confirmed during week 12 of pregnancy. This subject received cervical cold knife conization and cervical cerclage during her 16th week of pregnancy. The baby was carried to full term and delivered vaginally during week 39; both the subject and the baby survived. One subject was classified as HSIL on her cervical cytology test when she was 16 weeks pregnant. HPV tests and colposcopy were repeated during weeks 20 and 29; these showed persistent HPV-16 infection and stage IA1 squamous cell carcinoma, respectively. During week 34, we performed fetal lung maturation, cesarean section, extensive hysterectomy, para-aortic lymph node resection, right ovary suspension, and left ovary cryopreservation. Both the subject and the baby survived.

Two subjects had stage IA2 cervical cancer. Two years previously, one of these subjects underwent extended radical surgery to treat stage IA2 cervical cancer. This patient was pregnant with twins at the time of the study but subsequently experienced miscarriage during week 24 secondary to premature rupture of the fetal membranes and infection. The other subject was a 42 -year-old woman who never returned for follow-ups despite being classified as LSIL by cervical cytology tests during early pregnancy. Five months after delivery, the subject underwent cervical biopsy; pathological findings were consistent with stage IA2 adenosquamous carcinoma. This patient was then treated with a laparoscopic radical hysterectomy, bilateral salpingectomy, and pelvic lymph node resection.

The only subject with stage IB1 cervical cancer chose to terminate pregnancy and underwent radical hysterectomy, bilateral salpingectomy, and pelvic lymph node resection, during the 9th week of pregnancy. One subject never received a formal prenatal examination and visited the hospital for the first time during week 32 of pregnancy with a presenting concern of irregular vaginal bleeding. Stage IB3 cervical cancer was discovered. Cesarean section, extensive hysterectomy, pelvic lymphadenectomy, and bilateral ovarian suspension surgery were performed two weeks later. This patient was also prescribed postoperative docetaxel, two sessions of carboplatin chemotherapy, and supplementary radiotherapy.

One other subject had stage IIA1 cervical cancer and received two consecutive LSIL classifications by cervical cytology screening during pregnancy. A $2.5-\mathrm{cm}$ cervical lesion was noted after cesarean section. A tissue sample was removed and sent for pathological examination; this revealed cervical cancer. The subject received extensive hysterectomy, pelvic lymph node dissection, radiotherapy, and chemotherapy for three months after delivery.

We identified three cases of stage IIA2 cervical cancer. One subject was diagnosed with cervical cancer when she was 13 weeks pregnant. This patient received five cycles of TC chemotherapy while pregnant. Following cesarean section during week 35 , she received extensive hysterectomy, pelvic lymphadenectomy, and bilateral ovarian suspension surgery. Postoperative supplementary radiotherapy was also prescribed. Another subject that had stage IIA2 cervical cancer was diagnosed during week 18 of pregnancy. This patient subsequently developed intrauterine infection with a high fever during week 28. Cesarean section, along with extensive hysterectomy and pelvic cavity, was performed immediately. Supplementary radiotherapy was prescribed postpartum. The third case of stage IIA2 cervical cancer suffered from recurrent vaginal bleeding. Colposcopy at week 16 of pregnancy detected a cervical tumor. This patient received two cycles of TC chemotherapy, commencing in week 20. A healthy baby was delivered in week 33 by cesarean section. Subsequently, the patient underwent extensive hysterectomy and pelvic lymph node dissection.

The remaining two subjects were diagnosed with stage IIB cervical cancer. One patient was referred from another hospital at 30+ weeks of pregnancy for the evaluation and treatment of cervical cancer and treatment. This subject was a 42-year-old woman who had not received a formal prenatal examination. Following fetal lung maturation, cesarean section was performed during week 33 of pregnancy; the patient also received postoperative radiotherapy. The other subject had received a normal cervical cytology result but was confirmed to have endometrial adenocarcinoma following cesarean section. This patient underwent laparoscopic extra-fascial hysterectomy (Table 1).

Of the 14 subjects analyzed in this study, 12 subjects survived and two were lost to follow-up. Except for the two that were aborted, all newborns survived. Two newborns were lost to follow-up.

\section{Discussion}

Cervical cancer is one of the most common gynecological malignancies in China; a previous report stated that approximately 130,000 new cases of cervical cancer occur every year, including 50,000 deaths. ${ }^{4}$ Early 
Table I Patient Information

\begin{tabular}{|c|c|c|c|c|c|c|c|c|c|}
\hline Case & Age & Weeks & HPV & Stage & Pathology Result & Therapy & Term & $\begin{array}{l}\text { Patient } \\
\text { Outcome }\end{array}$ & $\begin{array}{l}\text { Infant } \\
\text { Outcome }\end{array}$ \\
\hline 1 & 28 & $\begin{array}{l}2 \text { year before } \\
\text { pregnant }\end{array}$ & Positive & lal & $\begin{array}{l}\text { Squamous cell } \\
\text { carcinoma }\end{array}$ & $\begin{array}{l}\text { CKC and NSD ( } 2 \\
\text { years) }\end{array}$ & 39 & Alive & Alive \\
\hline 2 & 36 & 13 & No & Illa2 & $\begin{array}{l}\text { Adenosquamous } \\
\text { carcinoma }\end{array}$ & $\mathrm{TC}+\mathrm{CS}+\mathrm{RH}+\mathrm{RT}(\mathrm{PP})$ & 35 & Alive & Alive \\
\hline 3 & 34 & $\begin{array}{l}2 \text { year before } \\
\text { pregnant }\end{array}$ & 6,11 & la2 & Adenocarcinoma & RT & 24 & Alive & Miscarrage \\
\hline 4 & 35 & 32 & 16 & IB3 & $\begin{array}{l}\text { Squamous cell } \\
\text { carcinoma }\end{array}$ & $\mathrm{CS}+\mathrm{RH}+\mathrm{RT}(\mathrm{PP})$ & 35 & Alive & Alive \\
\hline 5 & 24 & 18 & No & Ila2 & $\begin{array}{l}\text { Adenosquamous } \\
\text { carcinoma }\end{array}$ & $\begin{array}{l}\mathrm{CS} \_\mathrm{RH}+\mathrm{RT} \text { (after } \\
\text { operation) }\end{array}$ & 28 & Missing & Alive \\
\hline 6 & 30 & PP & 16 & lal & $\begin{array}{l}\text { Squamous cell } \\
\text { carcinoma }\end{array}$ & $\mathrm{CS}+\mathrm{RT}(\mathrm{PP})$ & 36 & Alive & Alive \\
\hline 7 & 36 & 7 & 18 & $|b|$ & Adenocarcinoma & $\mathrm{RH}+\mathrm{RT}$ & 9 & Alive & Miscarrage \\
\hline 8 & 42 & 30 & No & Ilb & $\begin{array}{l}\text { Squamous cell } \\
\text { carcinoma }\end{array}$ & $\begin{array}{l}\mathrm{CS}+\mathrm{RT} \text { (after } \\
\text { operation) }\end{array}$ & 33 & Missing & Alive \\
\hline 9 & 33 & 3 months PP & No & Ila I & $\begin{array}{l}\text { Squamous cell } \\
\text { carcinoma }\end{array}$ & $\begin{array}{l}\mathrm{CS}+\mathrm{RH}+\mathrm{CCRT} \text { (after } \\
\text { Operation) }\end{array}$ & 38 & Alive & Alive \\
\hline 10 & 42 & 5 months PP & No & la2 & $\begin{array}{l}\text { Adenosquamous } \\
\text { carcinoma }\end{array}$ & $\mathrm{NSD}+\mathrm{RH}(5$ month PP $)$ & 39 & Alive & Alive \\
\hline II & 36 & 16 & No & Ila2 & $\begin{array}{l}\text { Squamous cell } \\
\text { carcinoma }\end{array}$ & $\mathrm{TC}+\mathrm{CS}+\mathrm{RH}$ & 33 & Alive & Alive \\
\hline 12 & 33 & 12 & 16 & lal & $\begin{array}{l}\text { Squamous cell } \\
\text { carcinoma }\end{array}$ & $C K C+N S D$ & 39 & Alive & Alive \\
\hline 13 & 33 & 20 & 16 & lal & $\begin{array}{l}\text { Squamous cell } \\
\text { carcinoma }\end{array}$ & $\mathrm{CS}+\mathrm{RH}$ & 34 & Alive & Alive \\
\hline 14 & 36 & 36 & No & Ilb & $\begin{array}{l}\text { Endometrial } \\
\text { adenocarcinoma }\end{array}$ & $\begin{array}{l}\mathrm{CS}+\mathrm{RH}(\text { after } \\
\text { operation) }\end{array}$ & 39 & Alive & Alive \\
\hline
\end{tabular}

screening and treatment of cervical cancer during pregnancy could significantly improve the prognosis and survival of pregnant women and their newborns. ${ }^{3}$

Cervical cancer can be asymptomatic in pregnant women. The signs and symptoms of cervical cancer, such as increased levels of vaginal bleeding, may often be confused with early symptoms of pregnancy. In this study, visible cauliflower-shaped lesions were found to be more common in squamous cell carcinoma and adenosquamous carcinoma.

The first trimester of pregnancy is often the hardest. Cervical evaluation during the early weeks of pregnancy can be very challenging as patients tend to be more anxious, especially during cervical cytological tests. Thus, pelvic examinations and cervical cytology screening are often performed during the second trimester, usually between 12 to 20 weeks of pregnancy. Observation alone is sufficient for pregnant women who have a normal cervical appearance and underwent cervical cytology screening with normal results within a year. Cervical cytology screening usually performed for all pregnant women who took their last test more than one year previously. Pregnant women with a suspicious cervical appearance upon pelvic examination are normally tested for HPV. If a visible cauliflower-shaped tumor is detected, then colposcopyguided cervical biopsy is performed in order to acquire 
tissue samples for pathological examination. ${ }^{5}$ Most researchers agree that colposcopy can be carried out safely at any time during pregnancy. ${ }^{2,6,7}$ Cervical cancer has the second highest incidence of any female malignant tumor in China. This is a common gynecological malignancy that mostly affects females aged between 20 and 40 years. Cervical cancer is rarer in non-pregnant women than in pregnant women. It has been reported that the incidence of cervical cancer in pregnancy lies between $1 /$ 2000 and 1/10,000. ${ }^{3}$ Another study, carried out in China, estimated that the incidence of cervical cancer in pregnancy was $0.10 \%-0.1 \% .{ }^{1}$ Due to the special immune status of pregnancy, cervical cancer during pregnancy will not affect either maternal and fetal outcomes in the middle or late phases of pregnancy. Therefore, over recent years, controversy has developed with regards to whether cervical cancer during pregnancy requires the immediate termination of pregnancy. However, following the identification of cervical cancer, a significant number of pregnant women still wish to continue their pregnancy. Therefore, there is a clear need to improve the quality of life for women with cervical cancer during pregnancy. Consequently, there is a need to develop differential diagnoses and treatment strategies according to the period, age, and pregnancy requirements. In the past five years, with the widespread screening of cervical lesions, the incidence rate of cervical cancer in the closely screened population decreased significantly by, ${ }^{3}$ closely related to the awareness of cervical screening and the increase of HPV vaccination rate. According to China's national conditions, the Chinese Society of Obstetrics and Gynecology has also designated relevant guidelines for cervical cancer screening during pregnancy: women who have not participated in cervical cancer screening, especially those who have never been screened; Women in need of cervical cancer screening again; Cervical cancer screening should be performed at the time of pre-pregnancy examination or the first prenatal examination. The screening method based on cervical cytology will not threaten the mother and infant during the whole pregnancy. Patients with cervical cancer whose clinical symptoms and signs cannot be excluded should be directly referred to colposcopy or biopsy and diagnosed according to the pathological results. ASC-US: high-risk human papillomavirus (HPV) detection: negative cases were delayed to postpartum reexamination; Positive patients should be referred to colposcopy. Direct referral to colposcopy is also available. LSIL: referral to colposcopy. If the cervical cytology is ASC-US or LSIL and there is no suspicious clinical history and signs, cervical cancer screening can also be performed six weeks after delivery. The initial colposcopy evaluation was LSIL or histological LSIL (cin1), and postpartum reexamination was recommended. Cytological ASC-H, HSIL, and above, AGC, and above should be referred to as colposcopy. Beijing and Shanghai are the representatives of the first-tier cities in China. The prevention and screening of diseases are more common. The screening rate of TCT in the Beijing obstetrics department is 30.76\% (21951/ 71360 ), and that in Shanghai is $100 \%$. Beijing Obstetrics and gynecology hospital and Shanghai National Women's and Infants Hospital belong to the third class A hospitals, with advanced treatment experience.

Therefore, in the present study, we retrospectively analyzed the clinical data of 14 pregnancies complicated by cervical lesions managed by these two hospitals over the last five years. Then, we analyzed their clinical characteristics, examinations, and treatment methods; we also followed-up each patient with regard to their pregnancy outcome. Further treatment and nursing care of patients with cervical cancer in a subsequent pregnancy. ${ }^{5}$ Rarely, mild vaginal bleeding can occur following colposcopy or cervical cytology testing; however, this is easily controlled. Miscarriage following colposcopy and cervical cytology testing has never been reported. ${ }^{2}$

Of the 14 cases of pregnancy complicated by cervical cancer included in this study, four cases were classified as false negatives in the cytology screening test. All of these had been classified as normal or LSIL according to cytology reports, with positive HPV. This suggests that pregnant women with abnormal cytology results and a concurrent high-risk of HPV infection should be further evaluated by colposcopy-guided biopsy. Immunohistochemistry should be performed, when necessary, for cases of cervical adenocarcinoma or adenosquamous carcinoma that are difficult to diagnose. It is also possible that hormonal changes during pregnancy may accelerate the progression of cervical cancer, thus leading to contradictory screening data. Although some studies have stated that pregnancy does not accelerate the progression of cervical cancer, small sample sizes have limited the validity of previous work; thus, further research now needs to be carried out in larger sample sizes. ${ }^{8}$

The management of cervical cancer during pregnancy should be individualized and based on the stage of cancer, the number of gestational weeks, and the fertility requirements of the patient involved. 
For stages of cervical cancer up to stage IA2, cold knife conization represents a useful treatment option. If cold knife conization is performed during pregnancy, patients should be monitored closely every two months by colposcopy. Cold knife conization should then be repeated six to eight weeks postpartum. ${ }^{9}$ Other treatment options include abortion followed by cold knife conization, or extensive cervical resection. Patients should then be monitored every three to six months postoperatively. A pregnancy is considered safe if cervical evaluations yield normal results for two consecutive years posttreatment. Conservative treatment is also a potential option. Recent studies have shown that pregnancy does not accelerate the progression of cervical cancer. Furthermore, prolonging the number of gestational weeks to promote fetal lung maturity has no adverse effect on pregnant women with cervical cancer. ${ }^{9}$

For patients with stage IB-IIA2 cervical cancer, surgical treatment remains controversial when patients have no para-uterine invasion. Most previous research recommends conservative treatment with chemotherapy; this is relatively safe during pregnancy. Neoadjuvant chemotherapy and TC chemotherapy can also be selected. Patients should be followed up regularly afterwards. If the cervical cancer is stable, then a cesarean section can be performed after 34 weeks of pregnancy with cervical surgery and supplementary postpartum chemoradiotherapy. If the cancer progresses, the treatment plan should be adjusted accordingly.

Stage IIB cervical cancer is at risk for metastasis and diffusion. Thus, the termination of pregnancy, with prompt treatment, is recommended for patients who are in the first and second trimesters. If stage IIB cervical cancer is noted in the second and third trimesters, then enhancement of fetal lung maturation is administered followed by adjuvant radiotherapy and chemotherapy post-delivery. ${ }^{10}$

Stage III-IV cervical cancer is rarely reported in the published literature; we did not identify any cases of stage III-IV cervical cancer in this study. However, the general recommendation for such patients is the immediate termination of pregnancy with subsequent radiotherapy and chemotherapy.

As women achieve pregnancies at a more advanced age, and malignant forms of cervical lesions become increasingly more common in younger age groups, the incidence of pregnancy complicated by cervical cancer will inevitably increase. It is therefore vital that we develop preventative measures for cervical cancer. Nowadays, HPV vaccines, including 2, 4, and 9 valent vaccines, are widely applied in the general population. However, the safety profile of these vaccines when used in pregnant or nursing women has yet to be established. There is no evidence to suggest that HPV vaccines prevent cervical cancer during pregnancy. Moreover, there is no evidence as to whether HPV vaccines cause fetal malformation. Thus, the general guideline is not to use HPV vaccines when pregnant or nursing.

Surgical treatment and local surgery can be viable choices for patients with early stages of cervical cancer. However, the safety of cervical conization and cerclage for cervical cancer in pregnancy remains unclear. It is possible for us to select the initial timing and protocol for chemotherapy; however, the long-term complications still need to be clarified in a larger cohort.

Current follow-up protocols are limited to wellness screening and the routine follow-up of patients and newborns. Samples of umbilical cord blood and serum antibodies from newborns are not routinely studied or monitored. Thus, the incidence of cervical cancer among this population remains unpredictable and further investigation is required.

\section{Ethics Approval}

This study was performed in accordance with the Declaration of Helsinki of the World Medical Association and was approved by the Ethics Committee of Beijing Obstetrics and Gynecology Hospital Capital Medical University (Reference: NO: KY-2014-005).

\section{Informed Consent}

This study is an observational study that did not involve the sampling of human specimens, did not include the exposure of personal information, was approved by the ethics committee, and did not require informed consent from the patients involved.

\section{Funding}

Supported by Beijing Municipal Science and Technology Commission (D131100005313009).

\section{Disclosure}

None of the authors have any conflicts of interest to declare with regard to this research.

\section{References}

1. Nguyen C, Montz FJ, Bristow RE. Management of stage I cervical cancer in pregnancy. Obstet Gynecol Surv. 2000;55(10):633-643. doi:10.1097/00006254-200010000-00022 
2. Zhang C, Wang M. progress in screening and management of cervical intraepithelial neoplasia during pregnancy. J Pract Obstet Gynecol. 2007;23(9):517-519.

3. Yudong W, Lulu S. Screening for cervical cancer during pregnancy. Chin J Pract Gynecol Obstetr. 2016;5:421-425.

4. Ping L. 13 years of clinical epidemiology data evaluation of cervical cancer in mainland China. China J Pract Gynecol Obstetr. 2018;34 (1):41-45.

5. Lihui W, Yun Z, Xing X, et al. Expert consensus on the management of pregnancy complicate with cervical cancer. Chin J Obstetr Gynecol. 2018;19(2):190-192.

6. Zunyou Z, Yu P. Syphilis and social upheaval in China. New Engl J Med. 2010;16:1658-1661.
7. Ghanem KG. Review: neurosyphilis: a historical perspective and review. CNS Neurosci Ther. 2010;16(5):e157-e168. doi:10.1111/ j.1755-5949.2010.00183.x

8. Gonçalves CV, Duarte G, Costa JS, et al. Diagnosis and treatment of cervical cancer during pregnancy. Sao Paulo Med J. 2009;127 (6):359-365. doi:10.1590/S1516-31802009000600008

9. National Health and Family Planning Commission of the People's Republic of China. Guidelines for standardized diagnosis and treatment of cervical and precancerous lesions. Chin J Med Front. 2013;5 (8):37-46.

10. Lingling X, Zhongqiu L. Progress and clinical controversy of cervical cancer treatment during pregnancy. Chin J Fam Plan Gynecol. 2019;2:10-14.

\section{Publish your work in this journal}

Cancer Management and Research is an international, peer-reviewed open access journal focusing on cancer research and the optimal use of preventative and integrated treatment interventions to achieve improved outcomes, enhanced survival and quality of life for the cancer patient.
The manuscript management system is completely online and includes a very quick and fair peer-review system, which is all easy to use. Visit http://www.dovepress.com/testimonials.php to read real quotes from published authors. 